\title{
Crystal-field model study of the xenon hexafluoride molecule. III. Electronic transitions and band shapes
}

\author{
Sylvester Y. Wang and Lawrence L. Lohr, Jr. \\ Department of Chemistry, University of Michigan, Ann Arbor, Michigan.48104 \\ (Received 4 March 1974)

\begin{abstract}
The application of a two-electron crystal-field model to the electronic structure of xenon hexafluoride is extended to include the calculation of oscillator strengths for absorption transitions to the largely spin singlet and the largely spin triplet excited states. Band shapes are calculated in terms of their spectral moments by obtaining vibrational energies and wavefunctions for the mixed quadratic-quartic potential energy functions calculated from the crystal-field model. The key experimental features of the absorption spectrum of the vapor are reproduced, namely the pronounced red shift and the increased bandwidth with rising temperature. The over-all similarity of the vapor spectrum to that of the isovalent hexahalotellurate (IV) complexes in solids is noted. It is concluded that the experimental data of Claassen, Goodman, and Kim are compatible with the pseudo-Jahn-Teller model of Gillespie as developed by Bartell and Gavin and by Wang and Lohr, and that the data do not require the use of the electronic isomers model of Goodman, although the latter model is not
\end{abstract} \\ excluded.
}

\section{INTRODUCTION}

In a recent paper ${ }^{1}$ we outlined a two-electron crystal field model of the electronic structure of the $\mathrm{XeF}_{6}$ molecule and presented detailed numerical results in the form of potential energy curves for ground and excited electronic states. This was followed by another study ${ }^{2}$ in which comparisons were made between the $\mathrm{XeF}_{6}^{\prime} \mathrm{re}-$ sults and those for the related $\mathrm{Xe}$ (VI) systems $\mathrm{XeOF}_{4}$, $\mathrm{XeO}_{2} \mathrm{~F}_{2}, \mathrm{XeO}_{3}$, and $\mathrm{XeF}_{8}^{2-}$. We return now to $\mathrm{XeF}_{6}$ and consider the application of the results of the first study to the problems of the ultraviolet absorption spectrum of the gaseous state. An extremely interesting feature of the reported ${ }^{3}$ spectrum is the long "tail" of the first absorption band at approximately $3400 \AA\left(29400 \mathrm{~cm}^{-1}\right)$, with this tail not only extending well into visible (to about $4500 \AA$ ), but also showing a marked increase in absorbance upon increasing the sample temperature from 25 to $90{ }^{\circ} \mathrm{C}$. By contrast the absorbance decreases with increasing temperature in the spectral range 3000-3300 $\AA$. These and other features of the $\mathrm{XeF}_{6}$ spectrum, including Raman data, ${ }^{3}$ were interpreted by Claassen, Goodman, and Kim using an electronic isomers model, ${ }^{4}$ in which it is proposed that $X_{e} F_{6}$ vapor consists of a thermally equilibrated mixture of octahedral, spin-singlet ground state molecules and trigonally distorted (both prolate and oblate), spin-triplet excited state molecules. The estimated fraction in the ground state at room temperature $\left(298.1^{\circ} \mathrm{K}\right)$ is 0.388 , with a fraction 0.538 having an oblate structure at a nonvertical energy of 446 $\mathrm{cm}^{-1}$ and a fraction 0.074 having a prolate structure at a nonvertical energy of $1229 \mathrm{~cm}^{-1}$. The spectral changes with temperature are then related to changes in the fractions for each of the three isomers. Since the results of our crystal-field study offered little support for the electronic isomers model and instead tended to support the pseudo-Jahn-Teller model expressed by Bartell and Gavin $^{5}$ following the ideas of Gillespie, ${ }^{b}$ it is important to test our theoretical results to see if they are compatible with the spectral data. Specifically it will be recalled that our $\mathrm{XeF}_{6}$ potential energy curves in the $t_{2 g}$ bending space did not, for what we took to be reasonable choices of electronic parameters, give excited state
Jahn-Teller stabilizations of sufficient magnitude to produce spin-triplet isomers which could be thermally populated. Rather it is the instability of the ground state forward $t_{1 u}$ bending and/or stretching deformations, together with mixing between $t_{1 u}$ and other modes which is suggested to be the explanation for many of observed differences between $\mathrm{XeF}_{6}$ and other hexafluorides.

\section{ELECTRONIC CONSIDERATIONS}

We write wavefunctions for the electronic and nuclear degrees of freedom in the adiabatic form

$$
\Psi_{e, n}(q, Q)=\psi_{e}(q, Q) \phi_{n}(Q, v),
$$

where $q$ and $Q$ denote the sets of electronic and nuclear coordinates, respectively. The electronic factor $\psi_{e}$ is a solution of the Schrödinger equation with nuclei held fixed at a structure $Q$, which is not necessarily the equilibrium structure $Q_{0}$. The nuclear factor $\phi_{n}$ is a solution with quantum number $v$ of the Schrödinger equation containing the nuclear kinetic energy relative to the center of mass and an effective potential energy which is the total electronic energy $\epsilon(Q)$. Electric dipole transition moments, in the dipole length formulation, between states with different electronic factors may then be written

$$
\begin{gathered}
\left\langle\psi_{e}^{\prime}(q, Q) \phi_{n}^{\prime}\left(Q, v^{\prime}\right)|\mathrm{q}| \psi_{e}(q, Q) \phi_{n}(Q, v)\right\rangle \\
=\left\langle\phi_{n}^{\prime}\left(Q, v^{\prime}\right)\left|\mathbf{R}_{e}(Q)\right| \phi_{n}(Q, v)\right\rangle_{Q},
\end{gathered}
$$

where

$$
\mathbf{R}_{e}(Q)=\left\langle\psi_{e}^{\prime}(q, Q)|\mathbf{q}| \psi_{e}(q, Q)\right\rangle_{\alpha}
$$

and $q$ denotes the sum of the vector coordinates of the electrons. The subscripts $q$ and $Q$ on the matrix elements indicate the integration variable. Terms with the operator $\bar{Q}$ do not enter because of the orthogonality of $\psi_{e}$ and $\psi_{e}^{\prime}$. If $\mathbf{R}_{e}(Q)$ in (3) is taken to be independent of $Q$, say by assuming it to have at all $Q$ its value at $Q_{0}$, then

(2) reduces to

$$
\left\langle\Psi^{\prime}|\mathbf{q}| \Psi\right\rangle=\mathbf{R}_{e}\left(Q_{0}\right)\left\langle\phi_{n}^{\prime}\left(Q, v^{\prime}\right) \mid \phi_{n}(Q, v)\right\rangle_{Q},
$$

where the second factor in (4) is a vibrational overlap integral whose square is a Franck-Condon factor. 
TABLE I. State functions ${ }^{\mathrm{a}}$ for ${ }^{1} A_{1, \boldsymbol{f}}$ and ${ }^{1,3} T_{1 u}$ at $O_{h}$ symmetry.

\begin{tabular}{llllllllll}
\hline \hline $\begin{array}{l}\text { Parameter } \\
\text { set }^{\mathrm{b}}\end{array}$ & $\begin{array}{l}\Delta E \\
(5 s-5 p)\end{array}$ & $F_{1}$ & $F_{2}$ & $\zeta(5 p)$ & $a_{1}$ & $b_{1}$ & $c_{1}$ & $a_{2}$ & $b_{2}$ \\
\hline 1 & 3.5 & 1.91 & 0.32 & 1.70 & 0.9460 & 0.1366 & 0.2938 & 0.9709 & 0.2394 \\
2 & 3.5 & 1.00 & 0.17 & 0.87 & 0.9817 & 0.0386 & 0.1866 & 0.9728 & 0.2318 \\
3 & 3.5 & 0.70 & 0.11 & 0.87 & 0.9889 & 0.0299 & 0.1454 & 0.9567 & 2.2911 \\
$4^{\mathrm{c}}$ & 3.5 & 0.50 & 0.08 & 0.87 & 0.9936 & 0.0227 & 0.1103 & 0.9379 & 0.3470 \\
5 & 3.5 & 0.30 & 0.05 & 0.87 & 0.9974 & 0.0144 & 0.0702 & 0.9066 & 0.4221 \\
6 & 2.0 & 0.50 & 0.08 & 0.87 & 0.9802 & 0.0740 & 0.1835 & 0.9379 & 0.3470 \\
7 & 1.0 & 0.50 & 0.08 & 0.87 & 0.8655 & 0.3519 & 0.3565 & 0.9379 & 0.3470 \\
8 & 0.5 & 0.50 & 0.80 & 0.87 & 0.4631 & 0.7526 & 0.4681 & 0.9379 & 0.3470 \\
\hline
\end{tabular}

The functions are defined in Eq. (5) in terms of $a_{1}, b_{1}, c_{1}, a_{2}$ and $b_{2}$.

bAll four energy parameters in electron volts.

"The so-called "best" set of Ref. 1.

For $\mathrm{XeF}_{6}$ with assumed $O_{h}$ symmetry and considering only the 28 two-electron states associated with the $5 \mathrm{~s}$ $\left(a_{1 g}\right)$ and $5 p\left(t_{1 u}\right)$ orbitals, there are only two allowed electric dipole transitions from the ${ }^{1} S_{0}\left(5 s^{2}\right)$ ground state. These are to the ${ }^{1} P_{1}^{0}$ and ${ }^{3} P_{1}^{0}$ levels of the $5 s 5 p$ configuration, where the spin multiplicity labels are used simply to indicate the principal basis state in each case and are not meant to imply eigenstates of $\mathbf{S}^{2}$. The two excited multiplets can also be designated as ${ }^{1} T_{1 u}$ and ${ }^{3} T_{1 u}$, where the symmetry for the $J=1$ spin-orbit level is $T_{1 u}$ for each. We now evaluate $\mathbf{R}_{e}\left(Q=O_{h}\right)$ for the transitions using the wavefunctions

$$
\begin{aligned}
& { }^{1} A_{1 s}=a_{1}{ }^{1} S_{0}\left(5 s^{2}\right)+b_{1}{ }^{3} P_{0}\left(5 p^{2}\right)+c_{1}{ }^{1} S_{0}\left(5 p^{2}\right), \\
& { }^{1} T_{1 u}=a_{2}{ }^{1} P_{1}^{0}+b_{2}{ }^{3} P_{1}^{0}, \\
& { }^{3} T_{1 u}\left(T_{1 u}\right)=-b_{2}{ }^{1} P_{1}^{0}+a_{2}{ }^{3} P_{1}^{0},
\end{aligned}
$$

where the atomic designations are used here for $\left|L S J M_{J}\right\rangle$ basis functions. Using standard methods for constructing the latter functions from $\left|L S M_{L} M_{S}\right\rangle$ functions, the desired matrix elements are

$$
\begin{aligned}
& \left\langle{ }^{1} A_{1 \varepsilon}\left|\mathrm{q}=\mathrm{q}_{1}+\mathrm{q}_{2}\right|{ }^{1,3} T_{1 u}\right\rangle \\
& \quad=\langle 5 s|\mathrm{q}| 5 p\rangle\left[2^{1 / 2} a_{1} a_{2}-\left(\frac{2}{3}\right)^{1 / 2}\left(b_{1} b_{2}+c_{1} a_{2}\right)\right] \text { for }{ }^{1} T_{1 u}
\end{aligned}
$$
and

$$
\langle 5 s|\mathrm{q}| 5 p\rangle\left[-2^{1 / 2} a_{1} b_{2}-\left(\frac{2}{3}\right)^{1 / 2}\left(b_{1} a_{2}-c_{1} b_{2}\right)\right] \text { for }{ }^{3} T_{1 u} \text {. }
$$

Note that (5) and (6) consider spin-orbit mixings for both ground and excited states, as well as configuration interaction for the ground state. Thus the mixings are fully treated within the manifold of the 28 two-electron states. The magnitude of the one-electron integral $\langle 5 s|q| 5 p\rangle$ with $q$ equal to vector $r$ is $3^{-1 / 2}$ times that with scalar $r$ appearing as a parameter in the $l=1$ component of the crystal-field treatment for distorted $\mathrm{XeF}_{\mathrm{B}}$. Again we assume the value 2.055 a.u. (1.087 $\AA$ ) for $\langle 5 s|r| 5 p\rangle$ as computed ${ }^{1}$ from Gaussian basis set Xe atom SCF radial functions. The coefficients $a_{1}, b_{1}$, etc., appearing in (5) and (6) are given in Table I for 8 of the 20 electronic parameter sets we used. ${ }^{1}$

The oscillator strength $f$ may be written 7

$$
f=\left(8 \pi^{2} m \nu / h\right)\left|\mathbf{u} \cdot\left\langle{ }^{1} A_{1 \delta}|\mathbf{q}|^{1,3} T_{1 u}\right\rangle\right|^{2},
$$

where $\mathbf{u}$ is a unit vector in the direction of the polarization and where $m$ is the electron mass, $\nu$ the transition frequency, and $h$ is Planck's constant. The constant in
(7) is for oriented molecules with nondegenerate states, but the same value is obtained after averaging over orientation and polarization direction and considering the triple degeneracy of the final $J=1$ level. The transition moments (6) and resulting $f$ values (7) are given in Table II together with the computed $\Delta E$ values for the parameter sets the wavefunctions in Table $I$. We observe for parameter sets $1-7$ that the ratio of the $f$ values for the transitions to the mostly spin-singlet and mostly spin-triplet excited states is in the range of $25: 1$ to $10: 1$. An exception is set 8 , where the very small value of $0.5 \mathrm{eV}$ for the $\Delta E_{5 s-5 p}$ parameter leads to a ground state which is mostly ${ }^{3} P_{0}\left(5 p^{2}\right)$, making the $f$ value small for the transition to the mostly singlet state of $5 s 5 p$. For the assumed "best" parameter set the ratio of $f$ values is 11.2 , with the ratio of $\Delta E^{\prime}$ 's being $4.58 \mathrm{eV} / 2.74$ $\mathrm{eV}=1.67$. The reported ${ }^{3}$ absorption spectrum has maxima at $3.67,4.90$, and $5.50 \mathrm{eV}$, with maximum extinction coefficient $\epsilon_{\max }$ values of approximately 460, 2600, and 3160 , respectively. In addition there is possibly a shoulder at roughly $3.1 \mathrm{eV}$. It is tempting to suggest that the features at 3.1 and $3.67 \mathrm{eV}$ are components of ${ }^{3} T_{1 u}$ split by the low-symmetry field of a $C_{3 v}$ or $C_{4 v}$ structure, while the features at 4.90 and $5.50 \mathrm{eV}$, with nearly an order of magnitude greater intensity, are components of ${ }^{1} T_{1 u}$.

Such an assignment is similar to that made $^{8}$ for the

\begin{tabular}{|c|c|c|c|c|c|c|}
\hline \multirow{2}{*}{$\begin{array}{l}\text { Parameter } \\
\text { set }\end{array}$} & \multicolumn{3}{|c|}{${ }^{1} A_{1} \longrightarrow \longrightarrow{ }^{3} T_{1 u}$} & \multicolumn{3}{|c|}{${ }^{1} A_{1} \longrightarrow \longrightarrow{ }^{1} T_{1 \mu}$} \\
\hline & $\Delta E$ & $M E$ & $f$ & $\Delta E$ & $M E$ & $f$ \\
\hline 1 & 2.39 & -0.233 & 0.034 & 7.64 & 0.652 & 0.857 \\
\hline 2 & 2.76 & -0.199 & 0.029 & 5.51 & 0.750 & 0.809 \\
\hline 3 & 2.72 & -0.248 & 0.044 & 4.96 & 0.764 & 0.753 \\
\hline $4^{c}$ & 2.74 & -0.297 & 0.064 & 4.58 & 0.770 & 0.712 \\
\hline 5 & 2.72 & -0.365 & 0.095 & 4.26 & 0.767 & 0.660 \\
\hline 6 & 1.25 & -0.305 & 0.030 & 3.12 & 0.715 & 0.421 \\
\hline 7 & 0.44 & -0.372 & 0.016 & 2.32 & 0.487 & 0.145 \\
\hline 8 & 0.46 & -0.421 & 0.021 & 2.35 & 0.026 & 0.0003 \\
\hline
\end{tabular}
$\mathrm{TeCl}_{6}^{2-}$ and $\mathrm{TeBr}_{6}^{2-}$ complexes, which display absorption spectra strongly resembling that for gaseous $\mathrm{XeF}_{6}$. Specifically the absorption spectrum of the $\mathrm{TeCl}_{6}^{4-}$ complex in its ammonium and tetra- $n$-butyl ammonium salts

TABLE II. Excitation energies, a dipole matrix elements, ${ }^{b}$ and oscillator strengths for $O_{h}$ symmetry.

${ }^{2}$ Denoted by $\triangle E$ and given in electron volts.

${ }^{b}$ Denoted by $M E$ and given in angstroms.

"The so-called "best" set of Ref. 1 . 
was interpreted in terms of transitions from the ${ }^{1} A_{1 \mathrm{~g}}$ $\left(a_{1 g}^{2}\right)$ ground state to mostly spin-triplet levels of $a_{1 g} t_{1 u}$ at $3.05,3.17$, and $3.81 \mathrm{eV}$, with $\epsilon_{\max }$ values of $700-$ 3000 , and to mostly spin-singlet levels of $a_{1 \varepsilon} t_{1 u}$ at 4.08 , 4.27 , and $4.42 \mathrm{eV}$, with $\epsilon_{\max }$ values of $8000-20000$. Our estimate of the ratio of the integrated intensity of the singlet region to that of the triplet region is $3.5: 1$, based on the reported ${ }^{8}$ spectrum. The splittings of the triplet levels were assigned as arising in part from spin-orbit coupling and in part from a geometry change in going to the excited state, the latter cause also invoked to explain the singlet splittings. Several ligandto-metal $t_{1 u}$ orbital charge-transfer bands were reported at energies of $4.74 \mathrm{eV}$ and higher. Excitation energies in salts containing the $\mathrm{TeBr}_{6}^{4-}$ complex were found to be typically $0.5 \mathrm{eV}$ less than their $\mathrm{TeCl}_{6}^{4-}$ counterparts. Interestingly the change in the $\mathrm{TeCl}_{6}^{4-}$ spectrum in going from 18 to $300^{\circ} \mathrm{K}$ is largely an increase in the bandwidth and asymmetry, with the increased skewness to low energies producing a thermal red shift in the mean energy without causing a pronounced shift in the position of the band maxima. These spectral features resemble those observed ${ }^{3}$ for gaseous $\mathrm{XeF}_{6}$.

Dipole transitions to levels of the $5 s 5 p$ configuration with $J \neq 1$ can also occur if the molecular geometry is deformed from $O_{h}$ symmetry. Table III gives the irreducible representations for the various levels in static symmetries $O_{h}, C_{4 v}, C_{3 v}$, and $C_{2 v^{*}}$. These structures all occur in the $t_{1 u}$ deformation space, as previously described. ${ }^{1,5}$ While the transitions to certain components of $J=2$ become allowed, that to $J=0$ of $5 s 5 p$ does not. This strongly forbidden transition will, however, become allowed if the symmetry is further reduced to $C_{s}$ (that for $t_{1 u}$ space with on Cartesian component being zero) or to $C_{1}$ (that for general points in $t_{1 u}$ space). By contrast transitions become allowed in $C_{4 v}, C_{3 v}$, and $C_{2 v}$ to at least one low symmetry component of each $J$ level of each term of $5 p^{2}$.

Selection rules for vibronically induced transitions from the spin-orbit $A_{1 g}$ ground level are readily obtained and show that the allowed final levels for such a process are $A_{1 g}, E_{\xi}, T_{1 \xi}$, and $T_{2 g}$ for a $t_{1 u}$ mode; $A_{2 \xi}$, $E_{g}, T_{1 g}$, and $T_{2 g}$ for a $t_{2 u}$ mode; $A_{2 u}, E_{u}, T_{1 u}$, and $T_{2 u}$

TABLE III. Symmetry of energy levels in various symmetries.

\begin{tabular}{|c|c|c|c|c|c|c|}
\hline Configuration & Term & $J$ level & $o_{n}$ & $C_{4 \nu}{ }^{2}$ & $C_{3 v}{ }^{2}$ & $C_{2 v}^{b}$ \\
\hline $5 s^{2}$ & ${ }^{1} S$ & 0 & $A_{18}$ & $A_{1}$ & $A_{1}$ & $A_{1}$ \\
\hline \multirow[t]{5}{*}{$5 s 5 p$} & ${ }^{3} p^{0}$ & 0 & $A_{1 u}$ & $A_{2}$ & $A_{2}$ & $A_{2}$ \\
\hline & & 1 & $T_{1 u}$ & $A_{1}+E$ & $A_{1}+E$ & $A_{1}+B_{1}+B_{2}$ \\
\hline & & 2 & $E_{u}$ & $A_{2}+B_{2}$ & $E$ & $A_{2}+B_{2}$ \\
\hline & & & $T_{2 u}$ & $B_{1}+E$ & $A_{2}+E$ & $A_{1}+A_{2}+B_{1}$ \\
\hline & ${ }^{1} P^{0}$ & 1 & $T_{1 u}$ & $A_{1}+E$ & $A_{1}+E$ & $A_{1}+B_{1}+B_{2}$ \\
\hline \multirow[t]{7}{*}{$5 p^{2}$} & ${ }^{3} P$ & 0 & $A_{18}$ & $A_{1}$ & $A_{1}$ & $A_{1}$ \\
\hline & & 1 & $T_{1 g}$ & $A_{2}+E$ & $A_{2}+E$ & $A_{2}+B_{1}+B_{2}$ \\
\hline & & 2 & $E_{\mathrm{g}}$ & $A_{1}+B_{1}$ & $E$ & $A_{1}+B_{1}$ \\
\hline & & & $T_{2 \boldsymbol{g}}$ & $B_{2}+E$ & $A_{1}+E$ & $A_{1}+A_{2}+B_{2}$ \\
\hline & ${ }^{1} \boldsymbol{D}$ & 2 & $E_{8}$ & $A_{\perp}+B_{1}$ & & $A_{1}+B_{1}$ \\
\hline & & & $T_{2 \boldsymbol{r}}$ & $B_{2}+E$ & $A_{1}+E$ & $A_{1}+A_{2}+B_{2}$ \\
\hline & ${ }^{\mathrm{t}} S$ & 0 & $A_{1<}$ & $A_{1}$ & $A_{1}$ & $A_{1}$ \\
\hline
\end{tabular}

apipole transitions from $A_{1}$ are allowed to $A_{1}$ and $E$.

${ }^{v}$ Dipole transitions from $A_{1}$ are allowed to $A_{1}, B_{1}$, and $B_{2}$. for a $t_{2 \varepsilon}$ mode; and $T_{1 u}$ and $T_{2 u}$ for an $e_{g}$ mode. The $A_{1 u}$ symmetry (Table III) of the $J=0$ level of ${ }^{3} T_{1 u}$ does not appear in the preceding, again confirming the strongly forbidden character of the absorption transition to this level, which is the first excited level in our computed energy spectrum. Octahedral molecules $\mathrm{AB}_{6}$ are "vibrationally deficient" in that they do not possess modes of $t_{1 \mathrm{~g}}$ symmetry necessary to induce electric dipole transitions from $A_{1 g}$ states to $A_{1 u}$ states.

\section{POTENTIAL ENERGY SURFACES AND VIBRATIONAL WAVEFUNCTIONS}

The shapes of the electronic absorption bands are determined for allowed transition by the vibrational overlap integrals in (4). We now estimate their value in the three-dimensional $t_{1 u}$ bending space. Energy matrix elements are available $e^{9-12}$ for one-, two-, and three-dimensional quartic oscillators in terms of the corresponding harmonic oscillator wavefunctions as basis functions, and these have been used in studies ${ }^{13}$ of quartic and mixed quadratic-quartic molecular vibrations. However for the three-dimensional case of interest here, a different matrix needs to be diagonalized for each value of the vibrational angular momentum quantum number $l$ that is associated with a thermally populated level of the ground electronic state. Thus it is desirable to reduce the problem to an effective one-dimensional oscillator problem without an explicit dependence on the quantum number $l$. We have previously noted that the excited state potential energy curves in $t_{1 u}$ bending space are roughly quadratic, so that for these states we choose

$$
V(Q)=\frac{1}{2} k Q^{2}=\frac{1}{2} k\left(Q_{x}^{2}+Q_{y}^{2}+Q_{z}^{2}\right),
$$

where $Q_{x}, Q_{y}$, and $Q_{z}$ are the Cartesian components of the $t_{1 u}$ bending mode, and where the force constant $k$ is chosen to match the curvature at the origin of the computed curves. Wavefunctions for the problem defined by (8) may be written exactly in spherical polar or Cartesian coordinates. For the latter choice, the functions are a product of one-dimensional harmonic oscillator functions in the variable $Q_{x}, Q_{y}$, and $Q_{z}$.

There are several representations of the ground state potential energy curves which preserve various features of the calculated curves but which lend themselves to simple computer solutions. One such case is that given by

$$
\begin{aligned}
V(Q) & =-a Q^{2}+b\left(Q_{x}^{4}+Q_{y}^{4}+Q_{x}^{4}\right) \\
& =V\left(Q_{x}\right)+V\left(Q_{y}\right)+V\left(Q_{z}\right),
\end{aligned}
$$

where

$$
V\left(Q_{x}\right)=-a Q_{x}^{2}+b Q_{x}^{4},
$$

and

$$
Q^{2}=Q_{x}^{2}+Q_{y}^{2}+Q_{z}^{2}
$$

This potential, as pointed out ${ }^{5}$ by Bartell and Gavin, implies a very high barrier to pseudorotation in $t_{1 u}$ space. Specifically there is a minimum energy structure with $C_{3 v}$ symmetry at an energy of $-3 a^{2} / 4 b$ and a radial displacement of $(3 a / 2 b)^{1 / 2}$, a minimum energy structure with $C_{2 v}$ symmetry at an energy of $-a^{2} / 2 b$ and 


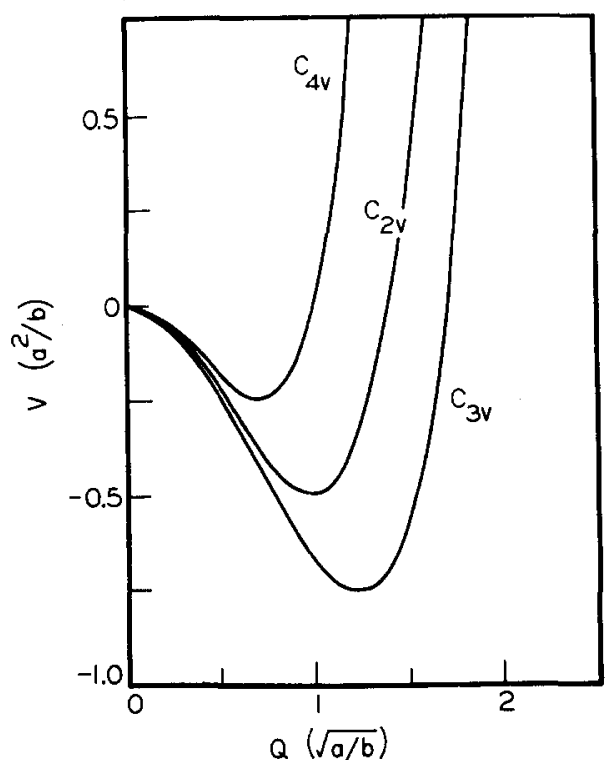

FIG. 1. Graphs of the mixed quadratic-quartic potential energy function given in Eqs. (9) and (10) for three directions in the three-dimensional $t_{1 u}$ bending space. The directions are [100] for $C_{4 v}$ symmetry, [110] for $C_{2 v}$ symmetry, and [111] for $C_{3 v}$ symmetry.

a radial displacement of $(a / b)^{1 / 2}$, and a minimum energy structure with $C_{4 v}$ symmetry at an energy of $-a^{2} / 4 b$ and a radial displacement of $(a / 2 b)^{1 / 2}$. Thus the stabiliza tion of a $C_{3 v}$ structure is 3 times that for a $C_{4 v}$ structure. Vertical energies at the radius of the $C_{3 v}$ minimum, $r=(3 a / 2 b)^{1 / 2}$, are even greater, namely $-3 a^{2} / 8 b$ for $C_{2 v}$ and $+3 a^{2} / 4 b$ for $C_{4 v}$ (Fig. 1).

The use of such a potential energy then reduces the problem to the solution of a one-dimensional Schrodinger equation

$$
\left[-(2 \mu)^{-1}\left(d^{2} / d Q_{x}^{2}\right)-a Q_{x}^{2}+b Q_{x}^{4}\right] \psi_{x}\left(Q_{x}\right)=E_{x} \psi_{x}\left(Q_{x}\right),
$$

where $\mu$ is the reduced mass. Identical equations hold in $Q_{y}$ and $Q_{z}$, so that

$$
\Psi\left(Q_{x}, Q_{y}, Q_{z}\right)=\psi_{x}\left(Q_{x}\right) \psi_{y}\left(Q_{y}\right) \psi_{z}\left(Q_{z}\right)
$$

and

$$
E=E_{x}+E_{y}+E_{z}
$$

Another simple case is that given by

$$
V(Q)=-a Q^{2}+b Q^{4}+l(l+1) / 2 \mu Q_{0}^{2},
$$

in which $l$ is the angular momentum quantum number and $Q_{0}$ is a fixed radius. Atomic units with $\hbar=1$ are used. This potential, which contains no pseudorotational barrier since the quartic term is isotropic, is rendered tractable by the assumption that the moment of pseudorotational inertia $\mu Q^{2}$ is approximately $\mu Q_{0}^{2}$, where the fixed radius $Q_{0}$ is usually that for the energy minimum. Thus this potential implies a rigid pseudorotor for which the Schrödinger equation may be written (in atomic units) as

$$
\left(-\frac{1}{2 \mu} \frac{d^{2}}{d Q^{2}}-a Q^{2}+b Q^{4}\right) P(Q)=\left(E-\frac{l(l+1)}{2 \mu Q_{0}^{2}}\right) P(Q)
$$

$$
=\left(\dot{E}-E_{\mathrm{rot}}\right) P(Q)=E_{\mathrm{vib}} P(Q)
$$

where $P(Q)=Q R(Q)$, in which $R(Q)$ is the usual radial function in the variable $Q$, and $E_{\text {rot }}$ and $E_{\text {vib }}$ are, respectively, the "rotational" and "vibrational" components of the energy. We must exercise caution in comparing (16), in which the range of $Q=\left(Q_{x}^{2}+Q_{y}^{2}+Q_{z}^{2}\right)^{1 / 2}$ is from 0 to $\infty$, to the standard one-dimensional form

$$
\left[-(2 \mu)^{-1}\left(d^{2} / d x^{2}\right)+V(x)\right] \psi(x)=E \psi(x)
$$

in which the range of $x$ is from $-\infty$ to $\infty$. If $V(x)$ is of even parity, the solutions $\psi(x)$ have either even or odd parity. The odd parity $\psi(x)$ necessarily have zero amplitude at the origin $(x=0)$. Such odd solutions and only such odd solutions of (17) are admissible as the solutions $P(Q)$ for (16), as $P(Q)=Q R(Q)$ must vanish as $Q-0$ because of the requirement that the usual radial function $R(Q)$ remain finite. Thus (16) may be solved ${ }^{12}$ by solving (17), in which $V(x)$ is taken as $-a x^{2}+b x^{4}$, for its odd solutions only. This leads to

$$
\Psi(Q, \theta, \phi)=[P(Q) / Q] Y_{l m}(\theta, \phi)
$$

and

$$
E=E_{\mathrm{vib}}+E_{\mathrm{rot}},
$$

where the $Y_{l m}$ factor is the standard normalized spherical harmonic in the angular variables of $t_{1 u}$ bending space.

A convenient computation procedure for obtaining solutions of the one-dimensional Schroddinger equations appearing in (17), which may stand for the types in (12) or (16), is to expand the solutions in a basis of harmonic oscillator eigenstates. This procedure ${ }^{14,15}$ has been frequently used before, for example in studies of hydrogen bonding, ${ }^{16}$ or ring deformations in bromocyclobutane and related compounds, ${ }^{17}$ and of intensity distributions for vibronically induced electronic transitions. ${ }^{18}$ This method is particularly practical when the potential energy function can be expressed as a short power series in the displacement variable. Details of the transformation of (17) to a dimensionless form are given in several references ${ }^{14-18}$ and are not repeated here. If $\psi_{i}(x)$ and $\psi_{j}(x)$ represent wavefunctions for the $i$ th level of the first potential energy function (electronic ground state curve) and the $j$ th level of the second potential energy function (electronic excited state curve), respectively, then

$$
\psi_{i}(x)=\sum_{v=0}^{v_{\max }} C_{v i} \phi_{v}(x)
$$

and

$$
\psi_{j}(x)=\sum_{v=0}^{v_{\max }} C_{v j} \phi_{v}(x),
$$

where $v$ is the usual harmonic oscillator (HO) quantum number, $\phi_{v}$ an HO eigenstate serving as a basis function, and the chosen number $m$ of HO basis functions is $v_{\max }+1$. The desired vibrational overlap integrals for use in (4) are then readily obtained by 


$$
\left\langle\psi_{i} \mid \psi_{j}\right\rangle=\sum_{v=0}^{v_{\max }} C_{i v} C_{j v},
$$

where use has been made of the fact that the same orthonormal basis set is used for describing levels of both the ground and excited state curves. The value of $m$ has typically been taken at 50 , implying $v_{\max }=49$, although some calculations were made with $m=30$.

\section{BAND SHAPES FOR ELECTRONIC EXCITATION}

As described in our study ${ }^{18}$ of band shapes for vibrationally induced electronic transitions, the energy distribution function $F(E, T)$ in which the energy $E$ is the distribution variable and the absolute temperature $T$ is a parameter, may be characterized by spectral semiinvariates $\Lambda_{k}(T)$ defined ${ }^{19}$ for our discontinuous distribution as follows:

$$
\Lambda_{1}(T) \equiv \bar{E}=\sum_{i, j} f_{i j}(T) \Delta E_{i j}
$$

and for $k \neq 1$,

$$
\Lambda_{k}(T) \equiv \overline{(E-\bar{E})^{k}}=\sum_{i, j} f_{i j}(T)\left(\Delta E_{i j}-\bar{E}\right)^{k} \text {. }
$$

In (22) $f_{i,}$ is the product of the square of the overlap integral in (21) and the Boltzmann factor for the vibrational level $i$ of the initial electronic state, while $\Delta E_{i j}$ $\equiv E_{j}-E_{i}$, the energy difference between the levels. The horizontal bars denote mean values.

One advantage of the use of spectral moments is that a conversion is readily made from the moments of a distribution in a single variable to those for a distribution, such as that for the three-dimensional $t_{1 u}$ bending mode, which is written as a convolution of a product of single variable distributions;

$$
F^{\prime}(E, T)=\left\langle F\left(E_{x}, T\right) F\left(E_{y}, T\right) F\left(E_{z}, T\right)\right\rangle,
$$

where the prime distinguishes the result from that for a single variable and where

$$
E=E_{x}+E_{y}+E_{z} \text {. }
$$

The angle brackets in (23) denote a convolution; that is, a summation over all $E_{x}, E_{y}$, and $E_{z}$ yielding a given value of $E$.

It is easily shown that for any number of variables that

$$
\begin{aligned}
\Lambda_{1}^{\prime} & =\sum_{i}\left(\Lambda_{1}\right)_{i}, \\
\Lambda_{2}^{\prime} & =\sum_{i}\left(\Lambda_{2}\right)_{i}, \\
\Lambda_{3}^{\prime} & =\sum_{i}\left(\Lambda_{3}\right)_{i}, \\
\Lambda_{4}^{\prime} & =\sum_{i}\left(\Lambda_{4}\right)_{i}+6 \sum_{i>j}\left(\Lambda_{2}\right)_{i}\left(\Lambda_{2}\right)_{j},
\end{aligned}
$$

where $i$ denotes the $i$ th variable and the parameter $T$ has been omitted for brevity. Expressions for higher moments are easily obtained and contain, like $\Lambda_{4}$ above, lower moments in the individual variables. It is not assumed in (25) that the sets of single variable moments $\left[\left(\Lambda_{k}\right)_{i}\right]$ are identical for each variable. For the $t_{1 u}$ bend, they are identical so that

$$
\begin{aligned}
& \Lambda_{1}^{\prime}=3 \Lambda_{1}, \\
& \Lambda_{2}^{\prime}=3 \Lambda_{2}, \\
& \Lambda_{3}^{\prime}=3 \Lambda_{3}, \\
& \Lambda_{4}^{\prime}=3 \Lambda_{4}+18 \Lambda_{2}^{2} .
\end{aligned}
$$

It should be recalled ${ }^{19}$ that the mean is given by $\Lambda_{1}$, the variance or the mean square deviation from the mean by $\Lambda_{2}$ (for a Gaussian distribution the half-width at halfheight $\Delta E_{1 / 2}$ is $\left.(2 \ln 2)^{1 / 2} \Lambda_{2}^{1 / 2}\right)$, the skewness $S$ by $\Lambda_{3} /$ $\Lambda_{2}^{3 / 2}$, and the kurtosis $K$ by $\left(\Lambda_{4} / \Lambda_{2}^{2}\right)-3$. Primed moments should be used if these measures are desired for the combined distribution, leading to the relationships $S^{\prime}=S / 3^{1 / 2}$ and $K^{\prime}=K / 3$.

In our earlier calculations ${ }^{1}$ of the potential energy surface in the $t_{1 u}$ bending space, we found by using the socalled "best" set of electronic parameters a miminum energy structure of $C_{3 v}$ symmetry characterized by fluorine displacements of $8.9^{\circ}$ along circular arcs with an assumed fixed $\mathrm{Xe}-\mathrm{F}$ distance of $1.89 \AA$ and by an energy stabilization of approximately $1860 \mathrm{~cm}^{-1}$ relative to an octahedral structure. The energy of a structure with $C_{4 v}$ symmetry was calculated to be only $160 \mathrm{~cm}^{-1}$ greater than that for the $C_{3 v}$ structure, although this pseudo rotational barrier was calculated to be much greater, namely $2015 \mathrm{~cm}^{-1}$, when coupling with the $t_{2 \xi}$ bending mode was included. The latter led to a $C_{3 v}$ symmetry structure $3710 \mathrm{~cm}^{-1}$ more stable than an $O_{h}$ symmetry structure and characterized by fluorine displacement of $13.6^{\circ}$ and $5.6^{\circ}$ in the $t_{1 u}$ and $t_{2 \zeta}$ bending spaces, respectively. We first consider the structure in pure $t_{1 u}$ space in connection with the potential energy function in (9) and (10). If $V_{0}$ is the well depth in ergs corresponding to $1860 \mathrm{~cm}^{-1}$ and $x_{0}$ the displacement of a fluorine atom in centimeters corresponding to $8.9^{\circ}$, then the potential energy coefficients for (9) or (10) are

$$
\begin{gathered}
a=2 V_{0} / x_{0}^{2}=8.55 \times 10^{4} \mathrm{erg} \mathrm{cm}^{-2}, \\
b=V_{0} / x_{0}^{4}=4.96 \times 10^{21} \mathrm{erg} \mathrm{cm}-
\end{gathered}
$$

in which $a$ and $b$ are chosen to fit the position and depth of the well. The potential defined by these coefficients can be used in the approximation defined by (15) and (16). To use (27) in $V(x)$ in (10), the coefficient $b$ of the quartic term should first be multiplied by 3 to express correctly the $C_{4 v}$ form of (10). As pointed out this implies a $C_{4 v}$ well depth only one-third as great as the $C_{3 v}$ depth of $1860 \mathrm{~cm}^{-1}$. Alternatively the direct use of $a$ and $b$ in (27) as a $C_{4 v}$ potential (10) implies a $C_{3 v}$ well depth of $3 \times 1860=5580 \mathrm{~cm}^{-1}$. While such large differences between the $C_{3 v}$ and $C_{4 v}$ minimal energies are not consistent with the results from our electronic model in pure $t_{1 u}$ bending space, they are much like our results in the mixed $t_{1 u}-t_{2 g}$ space so that we have used the coefficients $a$ and $b$ as well as the pair $a$ and $3 b$ to define crude model potentials (10) for a $C_{4 v}$ structure.

The calculation of vibrational energies, wavefunctions, and overlap integrals is actually carried out for a dimensionless Schrödinger equation which we have set up using a reference circular frequency of $\beta=4.77 \times 10^{13}$ 


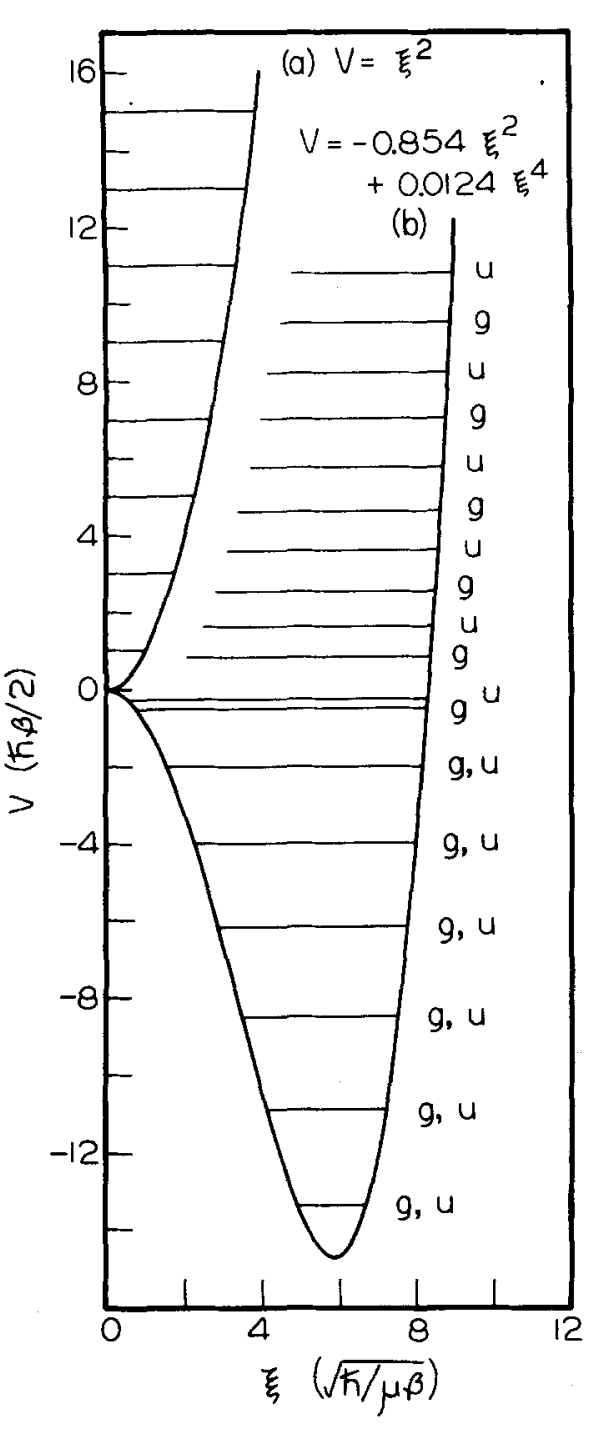

FIG. 2. Graphs of the excited state quadratic potential energy function (a) and the ground state mixed quadratic-quartic function (b), the latter as given in Eq. (28). Several energy eigenvalues are shown for each curve together with parity labels.

Levels labeled both $g$ and $u$ are essentially doubly degenerate:

$\mathrm{rad} \mathrm{sec}^{-1}$ and an assumed ${ }^{1,20}$ reduced mass of $53 \mathrm{amu}$. The value of $\beta$ was selected so that the excited state, assumed to be harmonic in $t_{1 u}$ space, is described by $V(\xi)$ $=\xi^{2}$, a dimensionless oscillator whose eigenfunctions are also the basis set for expansion for the ground state vibrational wavefunctions. For the latter state we have from (27) without multiplying $b$ by 3 ,

$$
V(\xi)=-0.854 \xi^{2}+0.0124 \xi^{4}
$$

where $\xi$ is the dimensionless variable related to centimeters by multiplying $\xi$ by $(\hbar / \mu \beta)^{1 / 2}=5.01 \times 10^{-10} \mathrm{~cm}$, and $V(\xi)$ is a dimensionless potential related to $\mathrm{cm}^{-1}$ by multiplying $V(\xi)$ by $\beta / 4 \pi c=126.5 \mathrm{~cm}^{-1}$, which is a factor corresponding to the zero point energy for the excited state harmonic oscillation. The excited state frequency is thus set by our choice of $\beta$ at $253 \mathrm{~cm}^{-1}$, a value similar to those for other hexafluorides. ${ }^{21}$ The implied ground state frequency obtained by using the curvature of (28) evaluated at its minimum of $\xi=5.87$ is 253 $\mathrm{cm}^{-1}$ times $(2 \times 0.854)^{1 / 2}$ or $331 \mathrm{~cm}^{-1}$. This follows from the fact that the curvature of $-a \xi^{2}+b \xi^{4}$ at the position of the minimum, $\xi=(a / 2 b)^{1 / 2}$, is $4 a$, while that of $\xi^{2}$ is 2 at al $\xi$. The actual spacings calculated for the anharmonic oscillator in (28) used directly as a $C_{4 v}$ potential (10) are $319,306,291,273,249,200 \mathrm{~cm}^{-1}$, etc. , starting from the lowest level. The calculated zero point energy in a single coordinate is $163 \mathrm{~cm}^{-1}$, more than half the first spacing and thus providing a measure of the anharmonicity. Ignored in the above list of spacings are the very small computed inversion splittings (less than $1 \mathrm{~cm}^{-1}$ ), so the lower levels are essential doubly degenerate.

The levels of the one-dimensional oscillator are shown in Fig. 2, together with those for the reference harmonic oscillator, the latter selected to match the chosen parabolic potential energy curve for the excited electronic state.

Band shape results for electronic absorption using 50 HO basis functions for the expansion of the eigenstates of (28) used in the one-dimensional Schrödinger equation (12) are given in Table IV as a function of temperature. The key features associated with increasing $T$ are:

(a) a pronounced red shift described by a decrease in $\Lambda_{1}^{\prime}$;

(b) a pronounced broadening associated with an increase in $\Lambda_{2}^{\prime}$, but tabulated as the half-width at halfheight for a Gaussian distribution;

(c) a change in the sign of the band asymmetry from positive (skewed to high $\Delta E$ ) to negative (skewed to low $\Delta E$ ) as given by the skewness $S^{\prime}$;

(d) an increase in the intensity in the "wings," as given by the kurtosis $K^{\prime}$, for temperatures up to about $450^{\circ} \mathrm{K}$, followed by a decrease in $K^{\prime}$.

All moments are the primed moments in (26), appropriate to a three-dimensional vibrational space. The above

TABLE IV. Band shapes using all states ${ }^{2}$ of $V=-0.854 \xi^{2}$ $+0.0124 \xi^{4}$.

\begin{tabular}{rclll}
\hline \hline$T\left({ }^{\circ} \mathrm{K}\right)$ & $\Delta E^{\mathrm{b}}\left(\mathrm{cm}^{-1}\right)$ & $\Delta E_{1 / 2}{ }^{\mathrm{c}}\left(\mathrm{cm}^{-1}\right)$ & \multicolumn{1}{c}{${ }^{\mathrm{d}} S^{\prime}$} & \multicolumn{1}{c}{${ }^{\mathrm{e}} K^{\prime}$} \\
\hline 0 & 0 & 1879 & 0.030 & -0.035 \\
50 & 0 & 1879 & 0.030 & -0.035 \\
100 & -11 & 1900 & 0.017 & -0.030 \\
150 & -57 & 1977 & -0.030 & -0.009 \\
200 & -129 & 2101 & -0.096 & 0.024 \\
250 & -224 & 2253 & -0.163 & 0.068 \\
300 & -334 & 2418 & -0.227 & 0.116 \\
350 & -459 & 2591 & -0.283 & 0.158 \\
400 & -596 & 2768 & -0.329 & 0.186 \\
450 & -740 & 2947 & -0.364 & 0.195 \\
500 & -888 & 3123 & -0.387 & 0.188 \\
550 & -1044 & 3293 & -0.401 & 0.167 \\
600 & -1200 & 3452 & -0.406 & 0.137 \\
\hline \hline
\end{tabular}

"Well depths and $1860 \mathrm{~cm}^{-1}$ for $C_{4 v}$ structure and $5580 \mathrm{~cm}^{-1}$ for $C_{3 v}$ structure. Excited state has $V=\xi^{2}$.

${ }^{\mathrm{b}} \Delta E=\Lambda_{1}^{\prime}(T)-\Lambda_{1}^{\prime}(0)$.

${ }^{c} \Delta E_{1 / 2}=(2 \ln 2)^{1 / 2}\left(\Lambda_{2}^{\prime}\right)^{1 / 2}$.

$\delta^{\prime}=\Lambda_{3}^{\prime} /\left(\Lambda_{2}^{\prime}\right)^{3 / 2}$.

' $K^{\prime}=\Lambda_{4}^{\prime} /\left(\Lambda_{2}^{\prime}\right)^{2}$. 


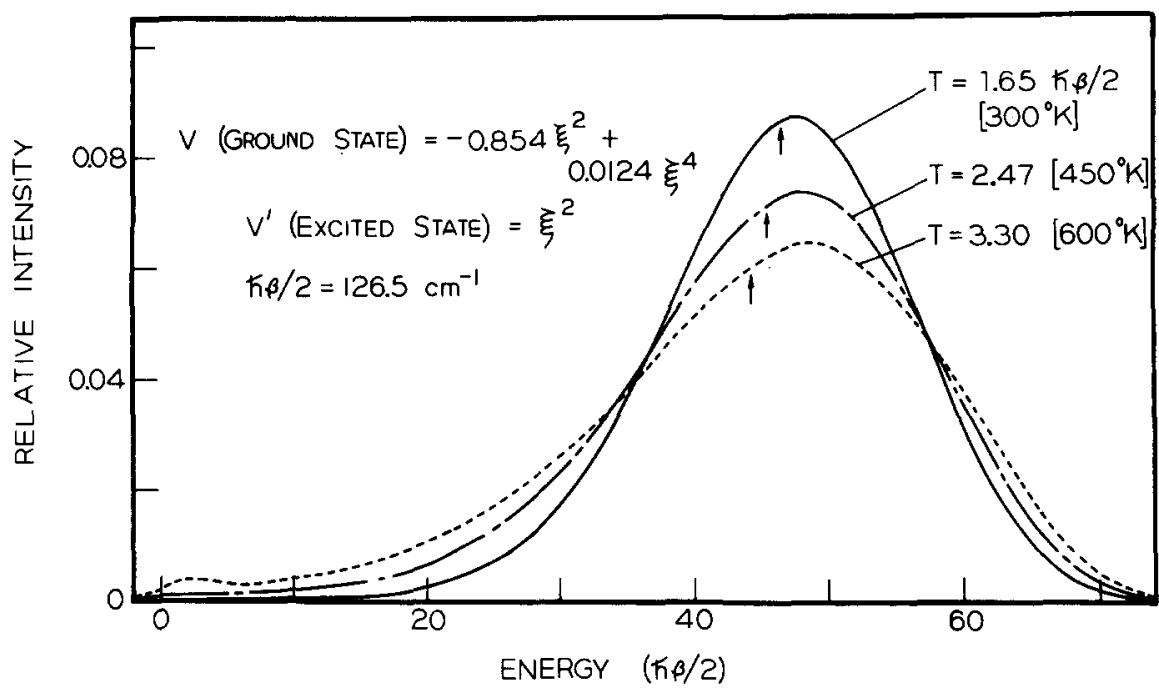

FIG. 3, Computed band shapes using the indicated potential energy functions and assuming that the vibrational degree of freedom is nondegenerate. The moments of these intensity distributions are the unprimed moments of Eq. (22). No electronic energy trace is included, so that energy scale is simply the vibrational component of the excitation energy, with zero corresponding to the vertical transition from the origin (not the minimum) of $V$ to the origin and the minimum of $V^{\prime}$. Smooth curves were drawn through computed points. changes with temperature result from changes in the Boltzmann populations for vibrational levels of the ground electronic state. The use of the relationships given in (26) eliminate the need for explicit consideration of the partition function in a three-dimensional space, it being sufficient to consider only a single dimension. The computed band shapes for a single $t_{1 u}$ coordinate are shown in Fig. 3 at temperatures of 300 , 450 , and $600{ }^{\circ} \mathrm{K}$. The only method we have for converting such a figure to one appropriate to a three-dimensional space is the method of moments, but this does not yield an attractive figure unless many moments are used. The thermal shift $\Delta E$ in the mean energy in the three-dimensional space is greater by a factor of 3 than that shown in Fig. 3, the increase in $\Delta E_{1 / 2}$ greater by a factor of $3^{1 / 2}, S$ smaller by a factor of $3^{1 / 2}$, and $K$ smaller by a factor of 3 .

The results given in Table $\mathrm{V}$ were obtained by solving (16) instead of (12), thus corresponding to an unhindered rather than a strongly hindered pseudorotor. The same potential (28) is used as before, so that there is still a very large radial barrier, leading to negligible vibrational amplitude near the origin. Thus using only odd-parity solutions of the one-dimensional Schrödinger

TABLE V. Band shapes using odd states ${ }^{\mathrm{a}}$ of $V=-0.854 \xi^{2}$ $+0.0124 \xi^{4}$.

\begin{tabular}{rccrr}
\hline$T\left({ }^{\circ} \mathrm{K}\right)$ & $\Delta E\left(\mathrm{~cm}^{-1}\right)$ & $\Delta E_{1 / 2}\left(\mathrm{~cm}^{-1}\right)$ & \multicolumn{1}{c}{$S^{\prime}$} & \multicolumn{1}{l}{$K^{\prime}$} \\
\hline 0 & 0 & 1085 & 0.052 & -0.105 \\
50 & 0 & 1085 & 0.052 & -0.109 \\
100 & -4 & 1096 & 0.028 & -0.090 \\
150 & -19 & 1141 & -0.052 & -0.027 \\
200 & -43 & 1212 & -0.166 & 0.072 \\
250 & -75 & 1301 & -0.281 & 0.195 \\
300 & -111 & 1394 & -0.386 & 0.318 \\
350 & -152 & 1494 & -0.478 & 0.417 \\
400 & -196 & 1594 & -0.549 & 0.477 \\
450 & -242 & 1693 & -0.603 & 0.486 \\
500 & -290 & 1789 & -0.639 & 0.456 \\
550 & -338 & 1882 & -0.660 & 0.396 \\
600 & -386 & 1971 & -0.669 & 0.312 \\
\hline \hline
\end{tabular}

${ }^{2}$ Well depths are $1860 \mathrm{~cm}^{-1}$ for both $C_{4 v}$ and $C_{3 v}$ structures. Excited state has $V=\xi^{2}$. equation (17) has a negligible effect on the one-dimensional moments. The states used are those labeled " $u$ " in Fig. 2. The vibrational overlap integrals do include angular variables, but these simply introduct Kronecker delta factors in the $l$ and $m$ quantum numbers. The results in Table $V$ differ from those in Table IV largely because the factors of 3 in (26) are now deleted. Thus $\Delta E$ in Table $\mathrm{V}$ is smaller by a factor of $3, \Delta E_{1 / 2}$ smaller by a factor of $3^{1 / 2}, S$ larger by a factor of $3^{1 / 2}$, and $K$ larger by a factor of 3 . The stabilization energy for the $C_{4 v}$ structure is again $1860 \mathrm{~cm}^{-1}$, but unlike the potential used in obtaining the results in Table IV, the stabilization energy of the $C_{3 v}$ structure is also $1860 \mathrm{~cm}^{-1}$, not $5580 \mathrm{~cm}^{-1}$.

Table VI presents results using a potential similar to (28), but obtained by multiplying the quartic coefficient by 3 , so that now the $C_{4 v}$ stabilization energy is $1860 / 3$ $=620 \mathrm{~cm}^{-1}$, while that for $C_{3 v}$ structure is $1860 \mathrm{~cm}^{-1}$. The trends in $\Delta E$ and $\Delta E_{1 / 2}$ are similar to those in Tables IV and V, while $S$ is becoming less negative, rather than more negative, at the higher temperatures, and $K$ is decreasing rather than increasing. The shallow $C_{4 v}$ well supports only four states, two even and two odd, below the radial barrier at $\xi=0$, so that states in which

TABLE VI. Band shapes using all states ${ }^{2}$ of $V=-0.854 \xi^{2}$ $+0.0372 \xi^{4}$

\begin{tabular}{rrlrr}
\hline \hline$T\left({ }^{\circ} \mathrm{K}\right)$ & $\Delta E\left(\mathrm{~cm}^{-1}\right)$ & $\Delta E_{1 / 2}\left(\mathrm{~cm}^{-1}\right)$ & \multicolumn{1}{c}{$S^{\prime}$} & $K^{\prime}$ \\
\hline 0 & 0 & 1090 & 0.087 & -0.072 \\
50 & -4 & 1088 & 0.093 & -0.078 \\
100 & -19 & 1112 & 0.054 & -0.055 \\
150 & -95 & 1188 & -0.052 & -0.010 \\
200 & -205 & 1290 & -0.137 & -0.014 \\
250 & -323 & 1391 & -0.176 & -0.060 \\
300 & -433 & 1479 & -0.184 & -0.118 \\
350 & -531 & 1552 & -0.177 & -0.170 \\
400 & -615 & 1614 & -0.164 & -0.213 \\
450 & -683 & 1665 & -0.148 & -0.248 \\
500 & -744 & 1708 & -0.133 & -0.276 \\
550 & -797 & 1744 & -0.119 & -0.299 \\
600 & -839 & 1775 & -0.107 & -0.317 \\
\hline \hline
\end{tabular}

${ }^{2}$ Well depths are $620 \mathrm{~cm}^{-1}$ for $C_{4 v}$ structure and $1860 \mathrm{~cm}^{-1}$ for $C_{3 v}$ structure. Excited state has $V=\xi^{2}$. 
TABLE VII. Band shapes using all states ${ }^{2}$ of $V=-0.722 \xi^{2}$ $+0.00444 \xi^{4}$.

\begin{tabular}{cclcc}
\hline$T\left({ }^{\circ} \mathrm{K}\right)$ & $\Delta E\left(\mathrm{~cm}^{-1}\right)$ & $\Delta E_{1 / 2}\left(\mathrm{~cm}^{-1}\right)$ & $S^{\prime}$ & $K^{\prime}$ \\
\hline 0 & 0 & 2536 & -0.143 & -0.063 \\
50 & -167 & 2514 & -0.145 & -0.060 \\
100 & -201 & 2524 & -0.152 & -0.052 \\
150 & -285 & 2591 & -0.194 & -0.010 \\
200 & -440 & 2719 & -0.260 & 0.056 \\
250 & -649 & 2886 & -0.329 & 0.124 \\
300 & -888 & 3073 & -0.389 & 0.184 \\
350 & -1150 & 3269 & -0.438 & 0.236 \\
400 & -1424 & 3470 & -0.479 & 0.284 \\
450 & -1708 & 3672 & -0.514 & 0.328 \\
500 & -1997 & 3876 & -0.544 & 0.369 \\
550 & -2293 & 4081 & -0.569 & 0.407 \\
600 & -2597 & 4286 & -0.592 & 0.441 \\
\hline
\end{tabular}

Well depths are $3710 \mathrm{~cm}^{-1}$ for $C_{4 v}$ structure and $11130 \mathrm{~cm}^{-1}$ for $C_{3 v}$ structure. Excited state has $V=\xi^{2}$.

the radial motion carries over the $O_{h}$ origin become populated.

The results in Table VII differ from the others in that they are based on a fitting of the potential energy curve in the mixed $t_{1 u}-t_{2 g}$ bending space. The stabilization energy of $3710 \mathrm{~cm}^{-1}$ for the $C_{3 v}$ structure at a fluorine displacement of $13.6^{\circ}$ along the $t_{1 u}$ coordinate leads to coefficients

$$
\begin{aligned}
& a=7.32 \times 10^{4} \mathrm{erg} \mathrm{cm}^{-2}, \\
& b=1.82 \times 10^{21} \mathrm{erg} \mathrm{cm}^{-4},
\end{aligned}
$$

analogous to (27). Conversion to dimensionless coefficients was made using the same value of $\beta$ as before, but with $\mu$ slightly larger, namely $53.8 \mathrm{amu}$. We obtain a potential

$$
V(\xi)=-0.722 \xi^{2}+0.00444 \xi^{4},
$$

in which $\xi$ is related to $x$ by multiplying $\xi$ by $4.98 \times 10^{-10}$ $\mathrm{cm}$. If the quartic coefficient is multiplied by 3 yielding 0.01332 , the resulting potential (10) implies stabilization energies of $3710 / 3=1237$ and $3710 \mathrm{~cm}^{-1}$ for $C_{4 v}$ and $C_{3 v}$ structures, respectively. Such a potential is close to that used in obtaining the Table IV results, so no calculations were performed. Direct use of (30) as (10) implies stabilization energies of 3710 and $3710 \times 3=11130$ $\mathrm{cm}^{-1}$ for $C_{4 v}$ and $C_{3 v}$ structures, respectively. Although this is an extreme case, the corresponding band shape parameters are given in Table VII and are much like those in Table IV except that the parameters show a greater sensitivity to temperature.

Throughout this section it has been assumed that the electronic transition moment (3) is independent of the nuclear displacement coordinates. This assumption does not hold for the large amplitudes of vibrational motion associated with thermally populated excited levels, particularly as the mixing of xenon $5 s$ and $5 p$ orbitals is directly related ${ }^{1}$ to the displacement in $t_{1 u}$ space. However as the $5 s$ to $5 p$ excitation is dipole allowed for $O_{h}$ symmetry, the variation of (3) with displacements in $t_{1 u}$ space is hopefully not as important as the vibrational overlap integrals in determining the band shapes. To the extent that the assumption of a constant electronic moment (3) holds, the results of this section are applicable to the transitions to the mostly spin-singlet and to the mostly spin-triplet excited states (see Sec. II). Thus identical band shapes are predicted for the two transitions, the intensities differing because of different electronic factors (6). The basic reason that the mean excitation energy shifts to the lower values with increasing temperature is that the direction of the anharmonicity in the mixed quadratic-quartic potentials (10) is such that the thermally averaged root-mean-square displacement in $t_{1 u}$ space decreases, so that the molecule is on the average closer to the origin of the curves in Fig. 1 or 2, resulting in a diminished excitation energy.

It should be noted that a complete treatment of the electronic band shapes would include the effects associated with changes in geometry and/or force constants for the remaining 12 modes of molecular vibration. While these changes are hopefully small for some of these modes, there is no basis for assuming that changes associated with the totally symmetric $a_{1 s}$ mode can be ignored. The resulting contribution to the spectral moments and their thermal variation can be directly incorporated in (25). We cannot use the crystal-field model as presently formulated to generate potential energy curves in $a_{1 g}$ or other bond stretching spaces, as it does not include a repulsion between $\mathrm{Xe}^{6+}$ and $\mathrm{F}^{1-}$ ions at short separations. However, our extended Hückel semi-empirical molecular orbital calculations ${ }^{22}$ for $\mathrm{XeF}_{6}$, similar to those reported ${ }^{23,24}$ for $\mathrm{XeF}_{2}$ and $\mathrm{XeF}_{4}$, suggest that the equilibrium bond length may be roughly $0.05 \AA$ shorter in the $a_{1 s} t_{1 u}$ excited orbital configuration than in the $a_{1 g}^{2}$ ground state configuration. Both the $a_{1 g}$ and the $t_{1 \mu}$ orbital are antibonding, with the fraction of $5 \mathrm{~s}$ character in the $a_{1 \varepsilon}$ orbital being roughly $28 \%$ at the calculated ground state bond length of $2.2 \AA$, and increasing with decreasing bond length, while the fraction of $5 p$ character in the $t_{1 u}$ orbital is roughly $75 \%$ at the same separation, but decreasing with decreasing bond length. Recent $a b$ initio calculations ${ }^{25}$ with a large Gaussian basis set yield $22 \% 5 s$ and $62 \% 5 p$ in the highest occupied $a_{1 g}$ and lowest occupied $t_{1 u}$ orbitals, respectively, at the observed bond length of $1.89 \AA$. The photoelectron spectral bandwidth ${ }^{2 B}$ is about $0.3 \mathrm{eV}$ at half-height, indicating an appreciable change in bond length accompanying the removal of an electron from the $a_{1 g}$ orbital. However we have not as yet attempted to estimate the contributions from the $a_{1 g}$ stretching mode to the spectral moments.

\section{SUMMARY}

It has been the object of this series of papers ${ }^{1,2}$ to use a crystal-field model to provide a semiquantitative basis for the model of the electronic structure of xenon hexafluoride proposed by Gillespie ${ }^{6}$ and developed by Bartell and Gavin, ${ }^{5}$ namely the stereochemical importance of the electron lone pair as manifested in the pseudo-JahnTeller deformations. The potential energy functions calculated from the crystal-field model are crude, but are hopefully representations of essential structural and spectral features. Our calculated intensity of distributions and their variations with temperature resemble those observed ${ }^{3}$ by Claassen, Goodman, and Kim, lend- 
ing support to the interpretation of the properties of xenon hexafluoride in terms of the pseudo-Jahn-Teller model, although the electronic isomers model of Good$\operatorname{man}^{4}$ cannot be excluded.

Note added in proof: A recent multiple-scattering $X \alpha$ study $^{27}$ of the electronic structure of $\mathrm{XeF}_{6}$ reported an orbital energy difference of $3.42 \mathrm{eV}$ between the unoccupied $t_{1 u}$ orbital and the highest occupied $a_{1 g}$ orbital of an assumed $O_{h}$ symmetry molecule. This value is close to our assumed "best choice" of $3.5 \mathrm{eV}$ for the $\Delta E_{5 s m 5 p}$ parameter. X-ray photoelectron spectral data ${ }^{28}$ have also been recently reported for $\mathrm{Xe}, \mathrm{XeF}_{2}, \mathrm{XeF}_{4}$, $\mathrm{XeF}_{6}$, and $\mathrm{XeOF}_{4}$. One conclusion is that the charge on the fluorine is about $-0.24 e$ in all of the molecules.

\section{ACKNOWLEDGMENTS}

The authors wish to thank Professor Lawrence Bartell for his very helpful suggestions and the University of Michigan Computation Center for the use of its facilities.

'S. Y. Wang and L. L. Lohr, Jr., J. Chem. Phys, 60, 3901 (1974).

${ }^{2}$ S. Y. Wang and L. L. Lohr, Jr., J. Chem. Phys. 60, 3916 (1974).

${ }^{3}$ H. H. Claassen, G. L. Goodman, and H. Kim, J. Chem. Phys. 56, 5042 (1972).

${ }^{4}$ G. L. Goodman, J. Chem. Phys. 56, 5038 (1972).

${ }^{5}$ L. S. Bartell and R. M. Gavin, Jr., J. Chem. Phys. 48, 2466 (1968); also see R. M. Gavin, Jr., and L. S. Bartell, J. Chem. Phys. 48, 2460 (1968).

${ }^{6}$ R. J. Gillespie, in Nobel-Gas Compounds, edited by H. H. Hyman (University of Chicago, Chicago, 1963), pp. 333-339.

${ }^{7}$ For example see J. W. Griffith, The Theory of Transition Metal Ions (Cambridge U. P., New York, 1961), pp. 41-57.

${ }^{8}$ D. A. Couch, C. J. Wilkins, and H. Gray, J. Am. Chem. Soc. 92, 307 (1970).

${ }^{9}$ W. H. Shaffer, Rev. Mod. Phys. 16, 245 (1944).

${ }^{10}$ H. H. Nielsen, Rev. Mod. Phys. 23, 90 (1951).
${ }^{11}$ S. Bell and P. A. Warsop, J. Mol. Spectrosc. 22, 360 (1967). ${ }^{12}$ S. Bell, R. Davidson, and P. A. Warsop, J. Phys. B 3, 113 and $123(1970)$.

${ }^{13}$ Some one-dimensional examples are trimethylene oxide [see S. I. Chan, T. R. Borgers, J. W. Russell, H. L. Strauss, and W. D. Gwinn, J. Chem. Phys. 44, 1103 (1961)] and cyclobutanone [see J. R. Durig and R, C. Lord, J. Chem. Phys. 45,61 (1966)].

${ }^{14}$ E. Heilbronner, Hs. H. Günthard, and R. Gerdil, Helv. Chim. Acta 39, 1171 (1956).

${ }^{15}$ T. Ueda and T. Shimanouchi, J. Chem. Phys. 47, 4042 (1967).

${ }^{16}$ R. L. Somorjai and D. F. Hornig, J. Chem. Phys. 36, 1980 (1962).

${ }^{17}$ W. G. Rothschild, J. Chem. Phys. 45, 1214 (1966).

${ }^{18}$ L. L. Lohr, Jr., J. Am, Chem. Soc. 92, 2210 (1970).

${ }^{19}$ See for example, R. von Mises, Mathematical Theory of Probability and Statistics (Academic, New York, 1964), pp. 112-154.

${ }^{20}$ For zero displacement along a $C_{t \nu}$ coordinate $\mu=4 m_{\mathrm{F}} m_{\mathrm{XeF}_{2}} /$ $M W=52.45 \mathrm{amu}$; the value of $53 \mathrm{amu}$ includes a correction for fluorine displacements of approximately $10^{\circ}$ along a $C_{4 v}$ coordinate.

${ }^{21}$ Some experimental values in $\mathrm{cm}^{-1}$ for $v_{4}$, the lower of the two $t_{1 u}$ frequencies, are $262\left(\mathrm{MoF}_{6}\right), 265\left(\mathrm{TcF}_{6}\right), 275\left(\mathrm{RuF}_{6}\right), 283$ $\left(\mathrm{RhF}_{6}\right), 325\left(\mathrm{TeF}_{6}\right), 258\left(\mathrm{WF}_{6}\right)$, and $257\left(\mathrm{ReF}_{6}\right)$. See B. Weinstock and G. L. Goodman, Adv. Chem. Phys. 9, 169 (1965).

${ }^{22}$ L. L. Lohr, Jr. (unpublished results).

${ }^{23}$ L. L. Lohr, Jr., and W. N. Lipscomb, J. Am. Chem. Soc. 85, 240 (1963).

${ }^{24}$ L. L. Lohr, Jr., and W. N. Lipscomb, in Noble-Gas Compounds, edited by H. H. Hyman (University of Chicago, Chicago, 1963), pp. 347-353.

${ }^{25}$ H. Basch, J. W. Moskowitz, C. Hollister, and D. Hankin, J. Chem. Phys. 55, 1922 (1971).

${ }^{26} \mathrm{C}$. R. Brundle, G. R. Jones, and H. Basch, J. Chem. Phys. 55, 1098 (1971); also see photoionization mass spectral data given by J. Berkowitz, W. A. Chupka, P. M. Guyon, J. H. Holloway, and R. Spohr, J. Phys. Chem. 75, 1461 (1971).

${ }^{27}$ E. W. Phillips, J. W. D. Connolly, and S. B. Trickey, Chem. Phys. Lett. 17, 203 (1972).

${ }^{28}$ T. X. Carroll, R. W. Shaw, Jr., T. D. Thomas, C. Kindle, and N. Bartlett, J. Am. Chem. Soc. 96, 1989 (1974). 